\title{
Realistic Approach of the Relations of Uncertainty of Heisenberg
}

\author{
Paul E. Sterian \\ Academic Center for Optical Engineering and Photonics, Faculty of Applied Sciences, \\ University POLITEHNICA of Bucharest, 060042 Bucharest, Romania \\ Correspondence should be addressed to Paul E. Sterian; paul.sterian@yahoo.com
}

Received 10 October 2012; Accepted 24 December 2012

Academic Editor: Bogdan Mitrica

Copyright (C) 2013 Paul E. Sterian. This is an open access article distributed under the Creative Commons Attribution License, which permits unrestricted use, distribution, and reproduction in any medium, provided the original work is properly cited.

Due to the requirements of the principle of causality in the theory of relativity, one cannot make a device for the simultaneous measuring of the canonical conjugate variables in the conjugate Fourier spaces. Instead of admitting that a particle's position and its conjugate momentum cannot be accurately measured at the same time, we consider the only probabilities which can be determined when working at subatomic level to be valid. On the other hand, based on Schwinger's action principle and using the quadridimensional form of the unitary transformation generator function of the quantum operators in the paper, the general form of the evolution equation for these operators is established. In the nonrelativistic case one obtains the Heisenberg's type evolution equations which can be particularized to derive Heisenberg's uncertainty relations. The analysis of the uncertainty relations as implicit evolution equations allows us to put into evidence the intrinsic nature of the correlation expressed by these equations in straight relations with the measuring process. The independence of the quantisation postulate from the causal evolution postulate of quantum mechanics is also put into discussion.

\section{Introduction}

A quantum mechanical principle according to Werner Heisenberg as it is shown in his scientific paper [1] which was published in 1927, states, in its most common form, that it is not possible to simultaneously determine the position and momentum of a particle: "The more precisely the position is determined, the less precisely the momentum is known in this instant, and vice versa." This concept is fundamental to understanding quantum mechanics, the science of quantum systems like atoms, electrons, photons, neutrinos, and generally, particles in subatomic physics. It says that not all properties of a quantum particle can be measured with unlimited accuracy [2-29]. Until now, this has often been justified by the explanation that every measurement has necessarily to disturb the quantum particle thus distorting the results of any further measurements. This principle has proved to be true concerning the particles, so that even modern computers and nuclear power plants have been realized taking into account the uncertainty principle and other concepts specific to the field of quantum mechanics, which became the way we are thinking and working [30-55].
This principle known as the Heisenberg uncertainty principle can be expressed as follows:

$$
\Delta x \Delta p_{x} \geq \frac{1}{2} \hbar
$$

where $\Delta x$ is the uncertainty in position, $\Delta p_{x}$ is the uncertainty in momentum, and $\hbar$ is the reduced Planck's constant. Heisenberg's original paper does not attempt to rigorously determine the exact quantity on the right side of the inequality, but rather uses physical argument to show that the uncertainty between conjugate quantum mechanical variables is approximately $\hbar[1]$.

After Heisenberg, two other scientists, H. P. Robertson and E. Schrödinger, developed his uncertainty principle. They expanded it in 1930, by adding other pairs of variables that cannot be simultaneously known, such as: energy and position, angular position and angular momentum. The uncertainty principle continues to be refined and brought up to date. 
Generally, Heisenberg's uncertainty principle, states that incompatible dynamic variables in relation to the measuring process, satisfy the relations:

$$
\begin{gathered}
\Delta x \cdot \Delta p_{x} \geq \hbar, \quad \Delta E \cdot \Delta t \geq \hbar, \\
\Delta \varphi \cdot \Delta J_{z} \geq \hbar, \quad \Delta \phi \cdot \Delta n \geq \hbar \text { and so on }
\end{gathered}
$$

thus leading to the following statement:

The product of the inaccuracies arising from simultaneous determination of two canonical conjugate variables is of the order of magnitude of Planck's constant.

The above relations can be written, as it is known in the form of commutation relationships, between corresponding incompatible observables according to the theorem: being given two hermitic operators $A$ and $B$ and their commutator $C$, one can demonstrate the relationship

$$
\Delta A \Delta B \geq \frac{1}{2}|\langle C\rangle|
$$

where

$$
\begin{aligned}
& \Delta A=\sqrt{\left\langle A^{2}\right\rangle-\langle A\rangle^{2}}, \\
& \Delta B=\sqrt{\left\langle B^{2}\right\rangle-\langle B\rangle^{2}} .
\end{aligned}
$$

No actual physical measurements can avoid the limitations given by these relationships.

The conclusions drawn from the study of the universality of particle-wave dualism in the context of relativistic causality, and other observations resulting from mathematical formalisms of quantum theory led the authors to a new approach of Heisenberg's uncertainty relationships as they put into question the possibility of simultaneous determination in the measurement process of the uncertainties involved by these relationships, showing the incompatibility of "simultaneity" with relativistic causality requirements and proposing a reformulation of the principle of uncertainty.

From the classical theory of wave propagation, it is known that the width of a wave packet $\Delta x$ involves against reciprocal Fourier transform spaces of a pair of signals, an "indeterminacy" relationships of type

$$
\Delta x \Delta k \geq 2 \pi
$$

where $\Delta k$ representing scattering of wave vectors.

Similarly, in the case of finite duration disturbances $\Delta t$, one can highlight relationships such as

$$
\Delta t \Delta \omega \geq 2 \pi
$$

against reciprocal Fourier transform spaces of a pair of signals, where $\Delta \omega$ indicating the disturbance spectral content.

In quantum theory, relations (5) and (6) can be written as the first two uncertainty relations (2) for both photons and substantial particles. Fourier transformed reciprocal relationships can be identified, also for signals defined on spaces canonical variables involved in other expressions (2) representing uncertainty relations. For classical dynamic systems case for the canonical conjugate variables, one can define classical Poisson brackets, while within the context of quantum systems, we get the quantum Poisson brackets.

The incompatible quantum observables are correlated with Heisenberg's uncertainty principle, but as we have shown [4] the proposed new interpretation of this principle in the context of the relativistic causality. Accordingly, we cannot consider simultaneous measurements of the canonical conjugate variables, because these variables correspond to the reciprocal Fourier spaces. The standard experiments, for example, the diffraction of the electron by a slit, are not suitable, for the simultaneous measurements of the quantum particle position and impulse as it is usually accepted because, through the measuring apparatus (which realise the Fourier transform of the system state function), the information is propagated at a finite speed. The uncertainty relations can be understood as statistical correlations between the results of the measurements effected on the dynamic conjugate variables in the reciprocal Fourier spaces.

From another point of view, the analytical expressions of quantum observables can be obtained by replacing the classical canonically conjugate variables within a symmetrised expression of the classical variable $A$, by their corresponding quantum-mechanical operators. In this way the measuring particularities of the fundamental observables $r$ and $p$ are transferred to more complex observables which can be reported to the measuring processus, compatible or incompatible. For the measurement of any group of two or more compatible or incompatible observables, the "laws" of the measuring apparatus previously presented in $[3,4]$ are general. In the paper, the author considers the uncertainty relations as implicit evolution equations so the intrinsic nature of the correlations expressed by the uncertainty principle is shown more precisely. The quantification postulate and the causal evolution postulate are not independent of each other, contrary to the different systems of axioms of the quantum mechanics. The principled role of the incompatible observables in the evolution of the quantum systems is more realistically put into evidence by the above interpretation of the uncertainty relations of Heisenberg's.

\section{Fourier Transformers in the Measuring Process}

Switching, within the measuring process, from the direct variable space to the canonical conjugate variable space which we will be denoted by Fourier space requires a physical data processing system. We will illustrate that by the help of a twodimensional optical Fourier transform.

Let $g(x, y)$ be a function of two independent variables $x$ and $y$, which can be the coordinates of a point in a plane. If the following conditions are met:

(i) $g(x, y)$ is completely integrated into the infinite plane $(x, y)$; 
(ii) $g(x, y)$ has a finite number of discontinuities and a finite number of maxima and minima in any finite rectangle;

(iii) $g(x, y)$ has no infinite discontinuities,

then we get the following relations:

$$
\begin{aligned}
G\left(f_{x}, f_{y}\right) & =F\{g(x, y)\} \\
& =\iint_{-\infty}^{+\infty} g(x, y) \exp \left[-i\left(\omega_{x} x+\omega_{y} y\right)\right] \mathrm{d} x \mathrm{~d} y
\end{aligned}
$$

respectively,

$$
\begin{aligned}
g(x, y) & =F\left\{G\left(f_{x}, f_{y}\right)\right\} \\
& =\iint_{-\infty}^{+\infty} G\left(f_{x}, f_{y}\right) \exp \left[i\left(\omega_{x} x+\omega_{y} y\right)\right] \mathrm{d} f_{x} \mathrm{~d} f_{y},
\end{aligned}
$$

defining the direct and inverse Fourier transforms of the function $g(x, y)$. The quantities $f_{x}=\omega_{x} / 2 \pi$ and $f_{y}=\omega_{y} / 2 \pi$ are spatial frequencies and $G\left(f_{x}, f_{y}\right)$ is the signal spectral function.

If the functions do not satisfy the conditions of Fourier transform, then we get the generalized Fourier transform.

It is known that an optical system suitable for a twodimensional Fourier transform may be a lens. If the focal plane $P_{1}$ lies above the signal function $g(x, y)$, at the back focal plane $P_{2}$ of the lens, a Fourier spectrum $G\left(f_{x}, f_{y}\right)$ of the signal $g(x, y)$ is displayed. In this plane, spatial frequencies are given by the following relations:

$$
\omega_{x}=-\frac{2 \pi x_{2}}{\lambda F}, \quad \omega_{y}=-\frac{2 \pi y_{2}}{\lambda F}
$$

where $F$ is the focal length of the lens and $\lambda$ the wavelength of the radiation used. Optical Fourier transform property of a lens can be analyzed using the Huygens-Fresnel diffraction formula [10].

\section{Experimental Setting of Heisenberg Uncertainty Relations}

Heisenberg's uncertainty relations are usually established on the basis of the experimental observations. It is well known in this regard that Heisenberg imagined an experience of "simultaneous" measuring of the electron position and momentum by using "ray microscope." Other examples of experimental facts from which analysis suggest that the existence of uncertainty relations is as follows:

(i) experience concerning diffraction of electrons through a slit;

(ii) experience concerning the deviation of a particle in a magnetic field;

(iii) compton collision processes of particles with a photon, and so forth.
From the experiments of this type we can conclude that there can be no question of simultaneous measurement of incompatible observables due to the finite speed of propagation of interactions.

Let us assume the following hypotheses derived from the study presented in the previous paragraphs:

(i) canonical conjugate variables, that is, incompatible variables belong to reciprocal Fourier spaces;

(ii) Fourier transforms assume the existence of physical systems as information processing;

(iii) the deduction of uncertainty relations experimentally by using physical systems that perform Fourier transforms to reveal two canonical conjugate spaces and which can thus perform measurements on quantities which are incompatible.

Necessary Conclusions. Due to finite speed of propagation of interactions, signal switching within physical system to perform Fourier transform has a finite duration.

Therefore, the sizes of the input and output of the system that makes Fourier transforms and conjugates canonical variables or its incompatible observables are considered to perform simultaneous measurements, in the ordinary sense of the formulation of Heisenberg's uncertainty relations.

Reflecting the uncertainty relations, the wave-particle dualism still remains true, but its content is not changing the principle of uncertainty with respect to a simultaneous measurement.

The cause and the effect cannot be measured simultaneously as a consequence of the used measurement device (Fourier transformer), through which the signals pass at a finite speed. Far from contradicting the principle of causality, uncertainty relations come to confirm it, because if we admit Einstein relativity principles when analyzing the measurement process, one must take into account the finite speed of propagation of interactions.

Let us consider, for example, a moving quantum particle, such as electron or photon through a slot machine that is performing the Fourier transform, due to diffraction phenomena. Signal function at slot plane is the wave function of the particle. Its Fourier transform is pulse wave function in space which may be considered a simultaneous determination of the electron coordinate passing through the slot.

According to the interpretation presented in this paper, it results in the impossibility of localizing inside the volume element of the phase space, of a quantum particle at a given time moment, because the measurements of position and momentum of a quantum particle cannot be simultaneously made.

\section{Uncertainty Relations as Quantum Evolution Equations}

The canonically conjugate variables being correlated by uncertainty relations one must pay more attention to the problem of the nature of these correlations.

Let $S$ be the action-integral operator and $\eta_{i}, \xi_{i}$, the canonically conjugate variables in the quadridimensional 
Minkovsky space. One considers $\eta_{i}=x_{1}, x_{2}, x_{3}, x_{4}=$ $i c t ; \xi_{i}=\mathbf{P}_{i}=m_{0} \mathbf{U}_{i}$, where $\mathbf{P}_{i}$ is the $i$ component of the 4 impulse $\left[\mathbf{P}=\mathbf{P}\left(\vec{p}_{r},(i / c) \mathbf{E}\right)\right], \mathbf{U}_{i}$-the $i$ component of the 4-velocity, E-the relativistic energy, and $\vec{p}_{r}$ - the relativistic impulse of the system.

According to Schwinger's action principle, the change of the action operator is given by the following expressions:

$$
\begin{aligned}
& \delta S_{12}=F_{2}-F_{1}, \\
& F=\delta S=\sum_{i=1}^{4} \xi_{i} \eta_{i} .
\end{aligned}
$$

$F$ being the generator function of the unitary transformation which describes the system evolution and the variations $\delta \eta_{i}$ refering to the complete evolution of the system.

The operators $Q$ are changed by unitary infinitesimal transformations of the form:

$$
U=1+i \alpha F^{\prime}=1+i F
$$

according to the following:

$$
Q \longrightarrow Q+\delta Q=Q+i[F, Q]
$$

that is,

$$
\delta Q=i[F, Q]
$$

where $\alpha$ is a real parameter and $F^{\prime}$ an hermitic operator. From expression (12), putting $\delta \eta_{i} \rightarrow \delta \eta_{i} / \hbar, \hbar$ being Planck constant, it results in

$$
\delta Q=\frac{i}{\hbar}\left[\sum \xi_{i} \delta \eta_{i}, Q\right]=\frac{i}{\hbar}\left[\sum \xi_{i}, Q\right] \delta \eta_{i},
$$

which represents the general form of the quantum evolution equations. The complete variation $\delta Q$ of the $Q$ operator can be writen under the following form:

$$
\delta Q=\sum_{i} \delta Q^{\left(\eta_{i}\right)}
$$

so that from (16) and (17), one obtains the evolution equations depending on the $\eta_{i}$ variables:

$$
\delta Q^{\left(\eta_{i}\right)}=\frac{i}{\hbar}\left[\xi_{i}, Q\right] \delta \eta_{i}
$$

which are of the Heisenberg operator evolution equations type.

Accordingly, for different $\eta_{i}$ we obtain the following:

$$
\delta Q^{\left(x_{i}\right)}=\frac{i}{\hbar}\left[p_{r_{x_{i}}}, Q\right] \delta x_{i}, \quad i=1,2,3,
$$

respectively,

$$
\delta Q^{\left(x_{4}\right)}=\frac{i}{\hbar}\left[\frac{i}{c} H, Q\right] \delta(i c t)
$$

or

$$
\delta Q^{(t)}=\frac{i}{\hbar}[-H, Q] \delta t
$$

From the classical point of view, the above equations take the following form:

$$
\begin{gathered}
\delta Q^{\left(x_{i}\right)}=\frac{i}{\hbar}\left[p_{i}, Q\right] \delta x_{i}, \quad i=1,2,3, \\
\delta Q^{(t)}=\frac{i}{\hbar}[-H, Q] \delta t .
\end{gathered}
$$

The time-evolution is described as

$$
\delta Q=-\dot{Q} \delta t
$$

and (23) becomes

$$
\dot{Q}=\frac{i}{\hbar}[H, Q],
$$

which are identical with Heisenberg's temporal evolution equation of the operators. Besides, (22) can be understood similarly as "space-evolution" equations of the operators.

Let us to consider the particular cases $\mathbf{Q}=x_{i}$; $t$. The result is that (22) and (23) are reduced to the Heisenberg's uncertainty relations:

$$
\left[p_{i}, x_{j}\right]=\frac{i}{\hbar} \delta_{i j}
$$

respectively,

$$
[H, t]=\frac{i}{\hbar} .
$$

Equations (26) and (27) can be considered as evolution equation with implicit dependence for the fundamental operators, the evolution of every variable being reported to the coordinate axe which corresponds to this very one. The hidden character of the evolution expressed by (26) and (27), directly put into evidence the intrinsic correlations between canonically conjugate variables, with straight implications in the measuring process. The intrinsic correlations between canonically conjugate variables are understood by the appearance of the wave functions to the reciprocal Fourier spaces [3]. These correlations imply the evolution since $\bar{p}=\bar{p}(t)$ and $\bar{r}=\bar{r}(t)$ but $\bar{p}(t) \neq 0$ so that $\bar{r}(t) \neq \bar{r}_{0}$. Therefore, the variables $p_{i}$ and $x_{i}$ cannot by principle be measured simultaneously.

The time interval of the Fourier transformation processing by the measuring apparatus to put into evidence the correlated $\bar{x}$-space and $\bar{p}$-space of the quantum system variables cannot be avoided. There are these intrinsic correlations which determine the imprecisions expressed by uncertainty relations and the impossibility to localize the quantum system inside of the elementary cell of the phase space expressed by the relation $\Delta p \Delta x=\hbar$.

\section{Discussions}

(a) The temporal evolution equation (25) shows the dependence of $Q$ on $t$ indirectly, via dependence of $Q$ on $H$, which is the conjugate variable corresponding to $t$, so that the dependence of $Q$ on $t$ is an implicit one, as it results from the intrinsic correlation between $t$ and $E$. If $\partial Q / \partial t=0, d Q / d t=0$ 
only if the observables $H$ and $Q$ are noncorrelated, that is, $[H, Q]=0$. In this case, $Q=$ ct. is a time-conservative variable.

From (22) the result is the dependence of $Q$ on $x_{i}$ via $p_{i}$, which is the variable a $x_{i}$-canonically conjugated. Consequently, if $\partial Q / \partial x_{i}=0$ and $\left[p_{i}, Q\right]=0\left(p_{i}\right.$ and $Q$ are noncorrelated), then $Q=$ ct., which means that $Q$ is space conservative. In conclusion, one can observe the symmetry between temporal development of the system (using $H$ operator) and spatial development of the system (using $\bar{p}$ operator).

(b) The interpretation of uncertainty Heisenberg's relations (26) and (27) as evolution equations with implicit dependence connects in some content the quantisation postulate and the causal evolution postulate of quantum mechanics, which appears in this context as being different forms of the same evolution principle.

(c) The results can be generalized for the analysis of any group of two canonically conjugate dynamical variables, the equations implying these variables showing an intrinsic correlation being implicitly present during the measuring process.

(d) One can assert the importance of the noncommutative operators which are principled, closed, and related to the evolution of the quantum system, contrary to the commutative ensembles of operators which define maximal the quantum states of the corresponding system.

\section{Conclusions}

(i) The "standard experiments" are not meant for simultaneous measurements of position and momentum of a quantum system, as it is usually admitted, being a consequence of the used measurement device (Fourier transformer), through which the signals pass having a finite speed.

(ii) Any quantum system is subject to uncertainty relations, proving its dual nature.

Due to the requirements of the principle of causality in the theory of relativity, one cannot make a device for simultaneous measuring of the canonical conjugate variables in the conjugate Fourier spaces, so that these interpretations of the measurements are nonsensical.

(iii) According to the interpretation we have presented in this paper, the result is the impossibility of localizing inside the volume element of the phase space of a quantum system at a given time, because the measurements of position and momentum of a quantum particle cannot be simultaneously performed.

(iv) Uncertainty relations must be regarded as statistical correlations between the results of measurements of the dynamic variables in Fourier conjugate spaces.

(v) Therefore, Heisenberg's uncertainty principle could be formulated as follows: "the results of measurements in reciprocal Fourier spaces of the canonical conjugate dynamic variables of the quantum systems are statistically correlated, the uncertainties product being of the order of magnitude of the constant of Planck."

(vi) The concordance between uncertainty relations interpretations based on that understanding of the measuring process and the implicit evolution equations derived in this paper is relevant for the coherence of the theory developed above.

(vii) The treatise of the uncertainly relations as implicit evolution equations allowed us to put into evidence the intrinsic nature of the correlation expressed by these equations in straight relations with the measuring process.

(viii) In the context of the above results, the quantisation postulate of quantum mechanics is not independent from the causal evolution postulate, that is, these two postulates are connected in the axiomatic development of the quantum theory. This shows the principled role of the incompatible observables in the evolution of the quantum systems.

\section{References}

[1] W. Heisenberg, "Über den anschaulichen Inhalt der quantentheoretischen Kinematik und Mechanik," Zeitschrift für Physik, vol. 43, no. 3-4, pp. 172-198, 1927.

[2] J. Schwinger, “The theory of quantized fields I," Physical Review, vol. 82, no. 6, pp. 914-927, 1951.

[3] P. E. Sterian, "Asupra simultaneităţii măsurărilor variabilelor conjugate canonic în relaţiile de incertitudine ale lui Heisenberg," Buletinul Institutului Politehnic București, vol. 44, no. 3, pp. 95-100, 1982 (Romanian).

[4] P. E. Sterian, "On the connection between Heisenberg's uncertainty relations and the evolution quantum equations," Buletinul Institutului Politehnic Bucureşti, vol. 46-47, pp. 43-45, 1984 (Romanian).

[5] K. Nakamura et al., "Review of particle phusics," Journal of Physics, vol. G37, Article ID 075021, 2010.

[6] E. Ştefănescu, A. R. Sterian, and P. Sterian, "Study on the fermion systems coupled by electric dipol interaction with the free electromagnetic field," in Advanced Laser Technologies 2004, vol. 5850 of Proceedings of SPIE, pp. 160-165, September 2004.

[7] B. Mitrica, Studiul dependentei directionale a fluxului de miuoni si posibiltati de testare ale modelelor de interactie hadronica [Ph.D. thesis], University of Bucharest, 2010.

[8] F. C. Maciuc, C. I. Stere, and A. R. P. Sterian, "The time evolution and multiple parameters variations in a time dependent numerical model applied for an $\mathrm{Er}^{3+}$ laser system," in Proceedings of the 11th International School on Quantum Electronics on Laser Physics and Applications, vol. 4397 of Proceedings of SPIE, pp. 84-88, September 2000.

[9] F. C. Maciuc, C. I. Stere, and A. R. P. Sterian, "Rate equations for an Erbium laser system, a numerical approach," in Proceedings of the 6th Conference on Optics (ROMOPTO '00), vol. 4430 of Proceedings of SPIE, pp. 136-146, September 2000.

[10] A. R. Sterian, Mecanică clasică, Printech Publishing House, Bucharest, Romania, 2010. 
[11] A. R. Sterian, "Computer modeling of the coherent optical amplifier and laser systems," in International Conference on Computational Science and Its Applications (ICCSA '07), vol. 4705 of Lecture Notes in Computer Science, pp. 436-449, Kuala Lumpur, Malaysia, August, 2007.

[12] L. Bogdan, A. R. Sterian, and D. Nedelcu, "Study of surface and bulk ultrasonic waves generated in solids numerical analysis," in ROMOPTO 2006: Eighth Conference on Optics, vol. 6785 of Proceedings of SPIE, September 2006.

[13] C. Cattani, "Fractional calculus and Shannon wavelets," Mathematical Problems in Engineering, vol. 2012, Article ID 502812, 26 pages, 2012.

[14] A. R. Sterian, Mecanica Cuantică, Omnia Univ. S.A.S.T. Publishing House, Braşov, Romania, 2003.

[15] C. Cattani, "La physique de la relativite en Belgique au debut du XX siecle," in Album Belgique, A. Laserra, Ed., pp. 131-150, P.I.E. Peter Lang, 2010.

[16] C. Cattani, "Harmonic wavelet approximation of random, fractal and high frequency signals," Telecommunication Systems, vol. 43, no. 3-4, pp. 207-217, 2010.

[17] B. Lazar, A. R. Sterian, and D. Nedelcu, "Ultrasonic waves generated by laser based heat sources in semitransparent solids," in ROMOPTO 2006: Eighth Conference on Optics, vol. 6785 of Proceedings of SPIE, pp. Z7851-Z7851, 2007.

[18] P. E. Sterian, Mecanica relativistă şi noţiuni de teoria gravitaţiei, Library of Congress, USA, Editura Tehnică, Bucharest, Romania, 1979.

[19] L. Landau, E. Lipsit, V. Beresteski, E. Lifsit, and L. Pitaievski, Théorie Quantique Relativiste, Editura Mir, Moscou, Russia, 1972.

[20] B. Mitrica, I. M. Brancus, H. Rebel et al., "Experimentally guided Monte Carlo calculations of the atmospheric muon and neutrino flux," Nuclear Physics B, vol. 151, pp. 295-298, 2006.

[21] C. Cattani, "Signorini cylindrical waves and Shannon wavelets," Advances in Numerical Analysis, vol. 2012, Article ID 731591, 24 pages, 2012.

[22] C. Cattani, "Harmonic wavelets towards the solution of nonlinear PDE," Computers and Mathematics with Applications, vol. 50, no. 8-9, pp. 1191-1210, 2005.

[23] C. Cattani and I. Bochicchio, "Wavelet analysis of bifurcation in a competition model," in Proceedings of the 7th International Conference on Computational Science (ICCS '07), vol. 4488 of Lecture Notes in Computer Science, pp. 990-996, Beijing, China, May 1997.

[24] A. M. Apostu, A. L. Chirosca, C. Gomoiu et al., "Results of underground dose rates using TL detectors and preliminary TL characteristics of irradiated salt rock," Romanian Reports on Physics, vol. 63, no. 1, pp. 220-225, 2011.

[25] D. A. Iordache, P. Sterian, F. Pop, and A. R. Sterian, "Complex computer simulations, numerical artifacts, and numerical phenomena," International Journal of Computers, Communications and Control, vol. 5, no. 5, pp. 744-754, 2010.

[26] E. N. Stefanescu, P. E. Sterian, and A. R. Sterian, "Fundamental interactions in dissipative quantum systems," The Hyperion Scientific Journal, vol. 1, no. 1, pp. 87-92, 2000.

[27] E. Stefanescu, A. Sandulescu, and P. Sterian, "Quantum tunneling through a dissipative barrier," in Proceedings of the 4th Conference in Optics (Romopto '94), vol. 2461 of Proceedings of SPIE, pp. 218-225, March 1995.

[28] A. Saftoiu, O. Sima, I. Lazanu et al., "Simulation of electromagnetic showers in salt performed with Geant4," Romanian Reports in Physics, vol. 64, no. 1, pp. 314-324, 2012.
[29] A. M. Badescu, A. Saftoiu, O. Fratu et al., "Radio technique for investigating high energy cosmic neutrinos," Romanian Reports in Physics, vol. 64, pp. 281-293, 2012.

[30] E. N. Stefanescu et al., "Study of the fermion system coupled by electric dipol interaction with the free electromagnetic field," in Proceedings of the Advanced Laser Tehnologies, A. Giardini, V. I. Konov, and V. I. Pustavoy, Eds., vol. 5850 of Proceeding of SPIE, pp. 160-165, 2005.

[31] A. R. Sterian, "Coherent radiation generation and amplification in Erbium doped systems," in Advances in Optical Amplifiers, P. Urquhart, Ed., InTech, Vienna, Austria, 2011.

[32] I. M. Brancus, B. Mitrica, G. Toma et al., "Primary energy estimation and mass discrimination by the EAS lateral charged particle distributions simulated for KASCADE-Grande," International Journal of Modern Physics A, vol. 20, no. 29, pp. 67846786, 2005.

[33] A. R. Sterian, Fizica atomului si a solidului, Printech Publishing House, Bucharest, Romania, 2010.

[34] C. M. Bowden and J. P. Dowling, "Near-dipole-dipole effects in dense media: generalized Maxwell-Bloch equations," Physical Review A, vol. 47, no. 2, pp. 1247-1251, 1993.

[35] S. Arad, V. Arad, I. Onica et al., "Stability study for a large cavern in salt rock from Slanic Prahova," Acta Physica Polonica B, vol. 41, no. 7, pp. 1789-1802, 2010.

[36] H. Rebel, O. Sima, A. Haungs, C. Manailescu, B. Mitrica, and C. Morariu, "The muon charge ratio in cosmic ray air showers," Journal of Physics G, vol. 35, no. 8, Article ID 085203, 2008.

[37] M. Dima, M. Dulea, E. Pauna et al., "The Quantgrid project (RO): quantum security in grid computing applications," Romanian Journal in Physics, vol. 54, no. 5-6, pp. 441-448, 2009.

[38] S. Pascoli and T. Schwetz, "Prospects for neutrino oscillation physics," Advances in High Energy Physics. In press.

[39] M. Dima, M. Dulea, D. Aranghel et al., "Classical and quantum communications in grid computing," Optoelectronics and Advanced Materials, Rapid Communications, vol. 4, no. 11, pp. 1840-1843, 2010.

[40] B. Mitrica, "Asymmetry of charge ratio for low energetic muons," in Exotic Nuclei and Nuclear/Particle Astrophysics (II): Proceedings of the Carpathian Summer School of Physics, vol. 972 of AIP Conference Proceedings, p. 500, 2008.

[41] R. Margineanu et al., "Traces of radioactive ${ }^{131}$ I in rainwater and milk samples in Romania," Environmental Research Letters, vol. 6, no. 3, Article ID 034011.

[42] B. Mitrica, A. Bercuci, I. M. Brancus et al., "Muon decay, a possibility for precise measurements of muon charge ratio in the low energy range $(<1 \mathrm{GeV} / \mathrm{C})$," in Proceedings of the International Workshop on New Applications of Nuclear Fission, pp. 190-192, Bucharest, Romania, September 2003.

[43] A. Sterian and P. Sterian, "Mathematical models of dissipative systems in quantum engineering," Mathematical Problems in Engineering, vol. 2012, Article ID 347674, 12 pages, 2012.

[44] B. Mitrica, R. Margineanu, S. Stoica et al., "A mobile detector for measurements of the atmospheric muon flux in underground sites," Nuclear Instruments and Methods in Physics Research A, vol. 654, no. 1, pp. 176-183, 2011.

[45] C. A. Simion, N. Pãunescu, N. Mocanu, R. Cãlin, S. Bercea, and B. Mitricã, "Ultra low radiation background LSC measurements in a salt mine: a feasibility study," Journal of Labelled Compounds \& Radiopharmaceuticals, vol. 53, no. 5-6, pp. 307-311, 2010.

[46] C. Morariu, C. Manailescu, H. Rebel, O. Sima, A. Haungs, and B. Mitrica, "The muon charge ratio in cosmic ray air showers," Nuclear Physics B, vol. 196, pp. 414-417, 2009. 
[47] A. Toma and A. Sterian, "Signal processing and sampling method for obtaining time series corresponding to higher order derivatives," Mathematical Problems in Engineering, vol. 2010, Article ID 913147, 9 pages, 2010.

[48] A. R. Sterian and F. C. Maciuc, "Numerical model of an EDFA based on rate equations," in Proceedings of the 12th International School on Quantum Electronics: Laser Physics and Applications, vol. 5226 of Proceedings of SPIE, pp. 74-78, 2002.

[49] B. Mitrica, "20 years of cosmic muons research performed in IFIN -HH," in Exotic Nuclei and Nuclear/Particle Astrophysics (IV): From Nuclei to Stars: Carpathian Summer School of Physics, vol. 1498 of AIP Conference Proceedings, pp. 291-303, Sinaia, Romania, 2012.

[50] B. Mitrica, M. Petcu, A. Saftoiu et al., "Investigation of cosmic ray muons with the WILLI detector compared with the predictions of theoretical models and with semi-analytical formulae," Nuclear Physics B, vol. 196, pp. 462-465, 2009.

[51] A. Bercuci, B. Mitrica, M. Petcu et al., "Various types of measurements for atmospheric muons performed with the WILLI detector," in Proceedings of the Carpathian Summer School of Physics on Exotic Nuclei and Nuclear/Particle Astrophysics, pp. 367-371, Mamaia-Constanta, Romania, June 2005.

[52] I. M. Brancus, B. Mitrica, A. Bercuci et al., "Monte Carlo simulations and semianalytical parameterisations of the atmospheric muon flux controlled by muon charge ratio measurements performed with WILLI detector," in Proceedings of the 29th International Cosmic Ray Conference, vol. 6, p. 229, Pune, India, 2005.

[53] C. Rosu, D. Manaila-Maximean, D. Donescu, S. Frunza, and A. R. Sterian, "Influence of polarizing electric fields on the electrical and optical properties of polymer-clay composite system," Modern Physics Letters B, vol. 24, no. 1, pp. 65-73, 2010.

[54] E. Stefanescu, P. Sterian, and A. R. Sterian, "The Lindblad dynamics of a Fermi system in a particle dissipative environment," in International Conference on Advanced Laser Technologies (ALT '02), vol. 5147 of Proceedings of SPIE, pp. 160-168, September 2002.

[55] I. Lazanu, A. Apostu, I. Brancus et al., "About the possibility to measure some standard model parameters and search for new physics with low energy neutrinos," Romanian Reports in Physics, vol. 64, pp. 24-32, 2012. 

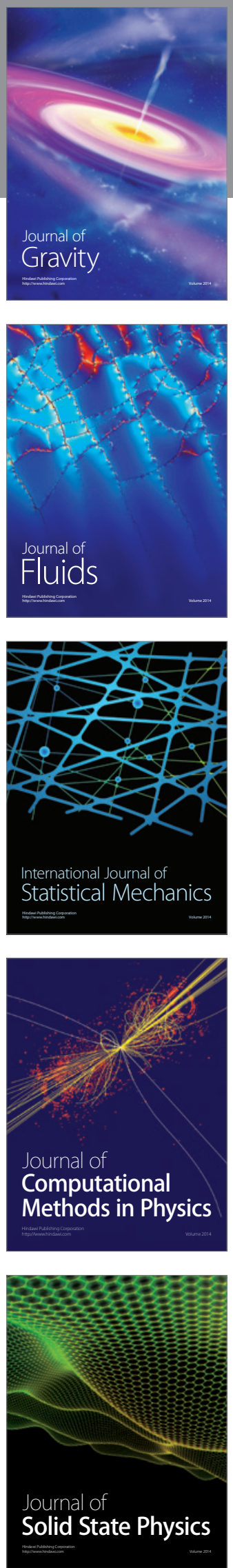

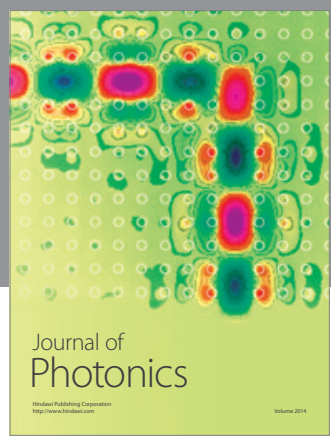

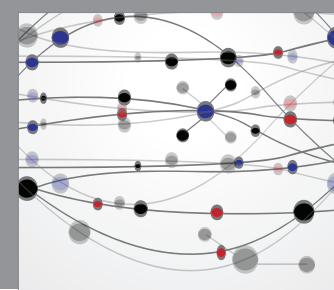

The Scientific World Journal

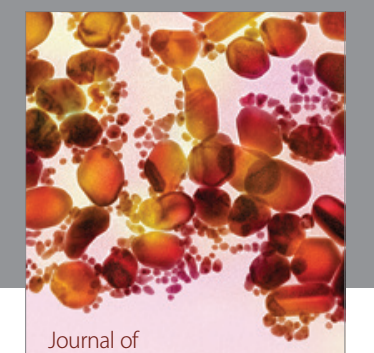

Soft Matter
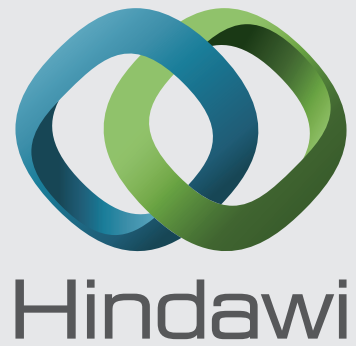

Submit your manuscripts at

http://www.hindawi.com
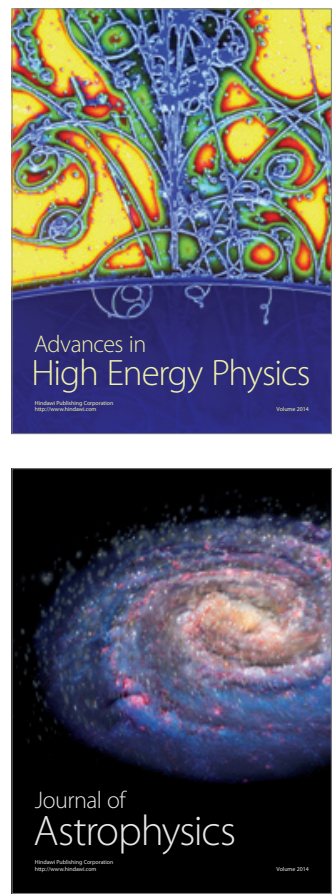
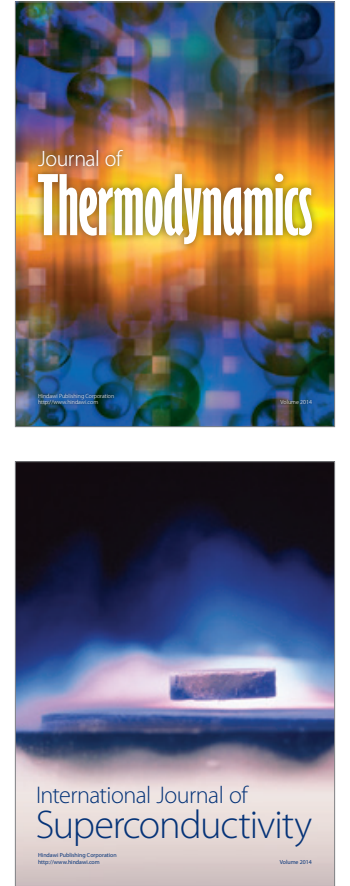
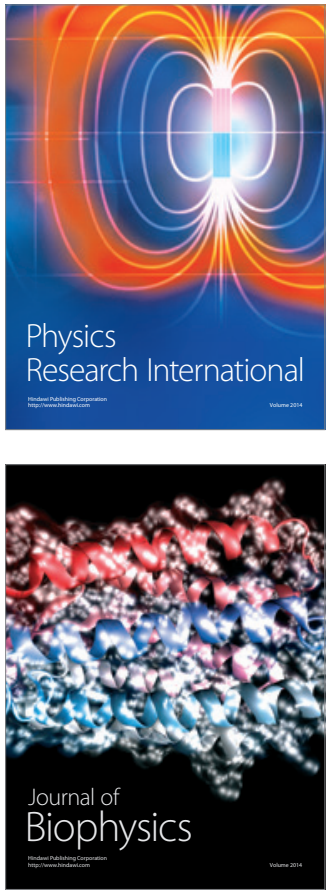
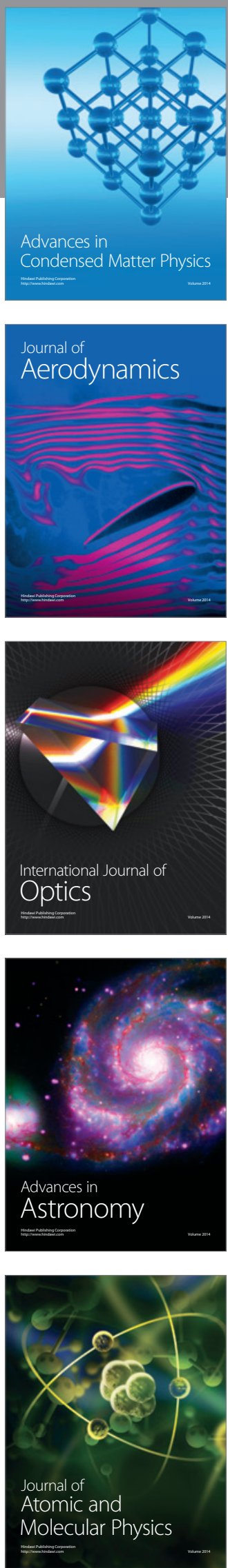